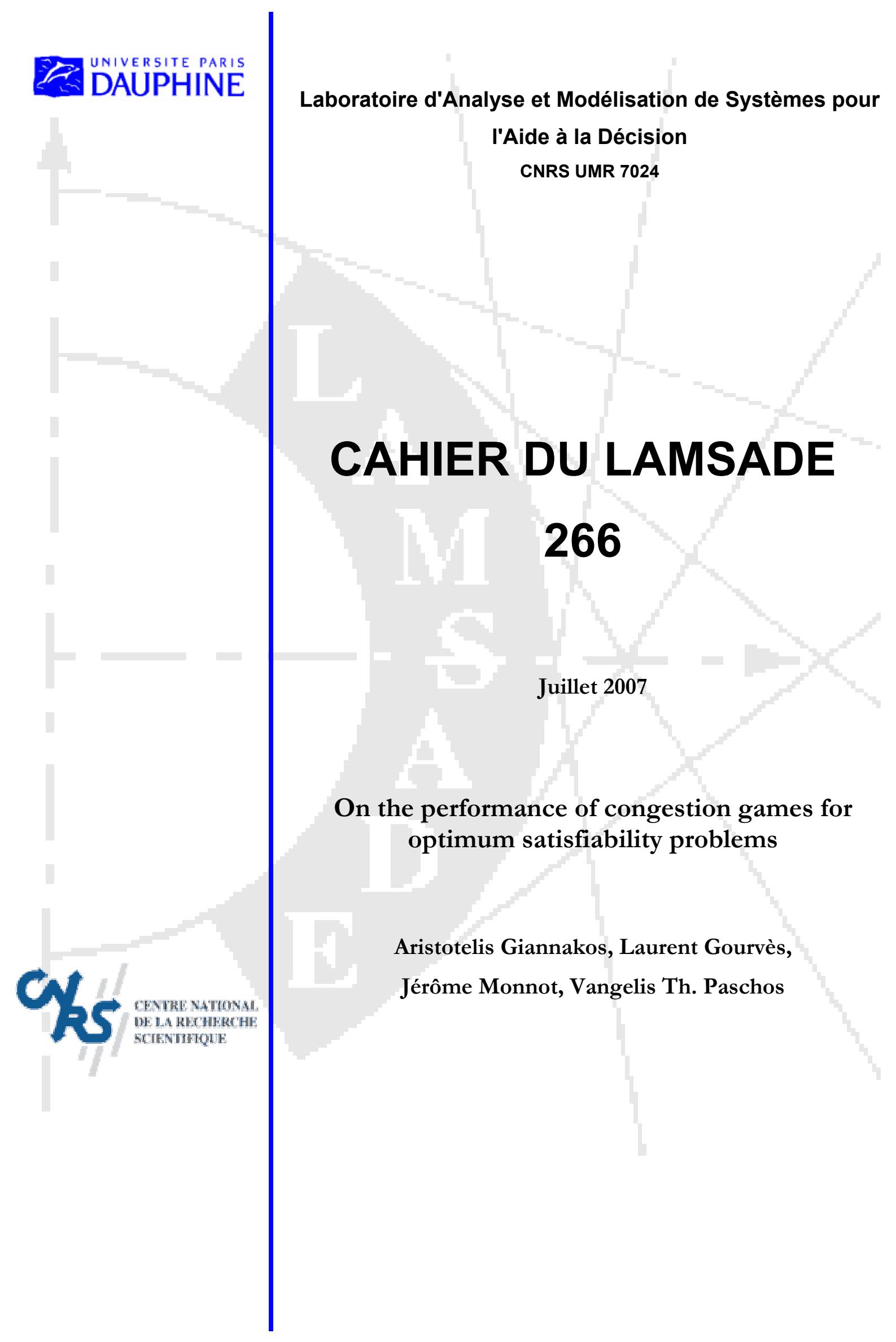




\title{
On the performance of congestion games for optimum satisfiability problems
}

\author{
Aristotelis Giannakos, Laurent Gourvès, Jérôme Monnot, and Vangelis Th. Paschos \\ LAMSADE, CNRS UMR 7024, Université de Paris-Dauphine, Paris, France \\ \{aristotelis.giannakos, laurent.gourves, monnot, paschos\}@lamsade.dauphine.fr
}

\begin{abstract}
We introduce and study a congestion game having MAX SAT as an underlying structure and show that its price of anarchy is $1 / 2$. The main result is a redesign of the game leading to an improved price of anarchy of $2 / 3$ from which we derive a non oblivious local search algorithm for MAX SAT with locality gap $2 / 3$. A similar congestion MIN SAT game is also studied.
\end{abstract}

keywords: price of anarchy, non oblivious local search, approximation algorithm, MAX SAT

\section{Introduction}

Starting from the seminal articles $[11,13,14]$, a lot of attention is paid to the performance of decentralized systems involving selfish users. Probably, the most extensively studied ones are congestion games [12,15] because they model central issues in networks. At the same time, the price of anarchy $(\mathrm{PoA})$ and the price of stability $(\mathrm{PoS})$ are certainly the most employed tools to analyze the performance of these games $[3,11,13]$.

A congestion game is a tuple $\left\langle N, M,\left(A_{i}\right)_{i \in N},\left(c_{j}\right)_{j \in M}\right\rangle$ where $N$ is the set of players, $M$ is the set of facilities, $A_{i} \subseteq 2^{M}$ is the set of strategies of player $i$ and $c_{j}$ is a cost function associated to facility $j$. In congestion games, a player's cost for using a facility depends only on the total number of players using this facility, and is independent of the player herself. A player's total cost is defined as the sum of the single costs over all facilities.

The PoA and the PoS are dominant tools to study the performance of decentralized systems $[3,11$, 13]. In minimization problems, the PoA (resp., the PoS) is the maximum (resp., the minimum) value that the ratio of the overall optimum to the cost of a Nash equilibrium (NE) can take over the set of all Nash equilibria. A NE is a combination of strategies, one for each agent, in which no agent has an incentive to unilaterally move away. Because Nash equilibria are known to deviate from the optimum in many optimization situations, the PoA captures the lack of coordination between independent agents while the PoS indicates how good a solution from which no agent will defect.

General congestion games make no particular assumption on the set of facilities; however, an extensive part of the literature deals with congestion games whose facilities sets are the edge sets of graphs (see for example $[11,14]$ on selfish routing in networks). In this paper, we introduce and study a congestion game associated to MAX SAT. The importance of this underlying structure does not need to be emphasized, since it is involved in numerous combinatorial optimization problems. Each clause is a facility and the players are the variables with strategy set $\{$ true, false $\}$ (playing true means selecting the clauses where her corresponding variable occurs unnegated, playing false means selecting the clauses where her corresponding variable occurs negated). Moreover, every clause/facility pays the variables/players a fraction $f(j)$ of its weight where $j$ denotes the number of players who satisfy it. Players rationally act in order to maximize their payments.

First, we discuss the question What is the price of anarchy and price of stability of the game? The next step is How can we reduce the price of anarchy? To do so, we use a powerful technique known as non oblivious local search $[1,10]$ which consists in using a specific cost function (i.e. different from the classical economic function) in a local search algorithm.

\section{Related work}

Articles on selfish routing in networks have mushroomed since the papers by $[11,14]$. Interestingly, congestion games provide the following unifying framework for such problems (often called network congestion games): we are given a graph $G=(V, E)$ where $E$ is the set of facilities. Each player chooses a 
path between a given source-destination pair of nodes in $V$. Each edge has a cost/latency function which depends on the number of players who use it. Each player selfishly selects her path in order to minimize her total cost/latency, i.e. the sum over all edges. The performance of the system is often measured by the average or maximum total cost/latency experimented by the players when they are at a Nash equilibrium.

In [11,7], the network consists of $m$ parallel links between a single source-destination couple of nodes. Players are weighted and the latency function associated to an edge is the sum of player's weights using it. The performance of the system is measured by the maximum total latency over all players. In $[4,5]$, the authors study linear general congestion games (not necessarily defined over a graph) with cost function of the form $c_{e}=a_{e} k+b_{e}$ where $k$ denotes the number of players using $e$ while $a_{e}$ and $b_{e}$ are positive coefficients. The performance of the system is both measured by the maximum and average total latency. In [3], Anshelevich et al. study a network design game where each edge is assigned a fixed cost. Each player buys/selects a path between her source and destination nodes. The cost of an edge is distributed over the players who select it. The social function is what the players collectively pay.

Algorithmic game theory and local search theory have several common points (see $[8,9]$ ). The locality gap (or approximation ratio) for a problem can be viewed as the PoA of a specific associated game. The well known fact that every congestion game admits a pure $\mathrm{NE}[12,15]$ can be interpreted in terms of local search theory: players, though separately guided by their self-interest follow a potential function and converge to a $\mathrm{NE}$ as a local search algorithm, following an economic function, converge to a local optimum.

A survey on MAX SAT, including local search algorithms, can be found in [2]. In particular, non oblivious local search has been successfully applied by Khanna et al. ([10]) who obtain a $\left(1-\frac{1}{2^{k}}\right)$ approximate algorithm for MAX E $k$-SAT (the restriction of MAX SAT to clauses with exactly $k$ literals). They use a 1-neighborhood (at most one variable is modified at a time) and a non oblivious function. When $k=2$, this function is $\frac{3}{2} \operatorname{cov}_{1}+2 \operatorname{cov}_{2}$ where $\operatorname{cov}_{i}$ denotes the number of clauses satisfied by exactly $i$ variables. With the classical (oblivious) function $\operatorname{cov}_{1}+\operatorname{cov}_{2}$, the locality gap is $2 / 3$ while the non oblivious function gives a 3/4-approximation algorithm. Unfortunately, Khanna et al. crucially use the fact that all clauses have exactly $k$ literals. Up to our knowledge, no extension of this approach to the MAX $k$-SAT problem (the restriction of MAX SAT to clauses with at most $k$ literals) is known. ${ }^{1}$

Changing the rule of a game in order to improve its PoA is not a new approach. In [6], Christodoulou et al., introduce the notion of coordination mechanism which attempts to redesign the system to reduce the PoA.

\section{Contribution and organization of the paper}

We introduce a congestion game (defined formally in Section 2) associated to the weighted MAX SAT problem where every clause/facility pays the variables/players a fraction $f(j)$ of its weight where $j$ denotes the number of players who satisfy it.

We first analyze the natural, so called fair, payment scheme where $f(j)=1 / j$ (the weight of a clause is evenly distributed to the variables who satisfy it) in Section 3. We undertake the same analysis in Section 4, using redesigned, in fact non oblivious, payment schemes and prove that the system shows improved performances.

Our results are summarized in the following table (results on the PoS are given in Section 5).

\begin{tabular}{|c|c|c|c|c|}
\cline { 2 - 5 } \multicolumn{1}{c|}{} & \multicolumn{2}{c|}{ MAX E $k-$ SAT } & \multicolumn{2}{l|}{ MAX $k-$ SAT } \\
\cline { 2 - 5 } \multicolumn{1}{c|}{} & PoA & PoS & PoA & PoS \\
\hline Fair & $\frac{k}{k+1}$ & $\frac{k}{k+1}$ & $\frac{k}{2 k-1}$ & $\leq \frac{k+1}{2 k}$ \\
\hline Non oblivious & $1-\frac{1}{2^{k}}$ & $1-\frac{1}{2^{k}}$ & $2 / 3$ & $2 / 3$ \\
\hline
\end{tabular}

Note that the given ratios cannot be improved under the considered payment scheme. Interestingly, we derive a $2 / 3$-approximate non oblivious local search algorithm using the 1 -neighborhood for the MAX $k-$ SAT problem. Finally, Section 6 is devoted to a game associated to MIN $k-$ SAT. We prove that its PoA, under the fair payment scheme, is equal to $k$ and that no non oblivious payment scheme can reduce it. Nevertheless, the PoS is equal to the $k^{t h}$ harmonic number $H(k)$ (even for MIN E $k$-SAT).

\footnotetext{
${ }^{1}$ It is not difficult to see that the locality gap of the MAX $k$-SAT problem is $1 / 2$ if we use the 1-neighborhood and the classical economic function, even for $k=2$.
} 
As done in [3-6] we only consider pure strategy Nash equilibria (all players deterministically choose between true or false). Thus, we restrict our analysis to the pure PoA and pure PoS of the MAX SAT and MIN SAT games.

\section{Definitions}

\subsection{MAX $k$ - SAT and MIN $k-$ SAT}

Let $X=\left\{x_{1}, \ldots, x_{n}\right\}$ be a set of boolean variables. A literal in $X$ is either a boolean variable $x_{i}$, or its negation $\overline{x_{i}}$, for some $1 \leq i \leq n$. A clause on $X$ is a disjunction of literals in $X$. The size of a clause $C$, i.e., the number of literals that $C$ contains, is denoted by $\sigma(C)$. An instance $I=(X, \mathcal{C}, w)$ of sat consists of a set of variables $X=\left\{x_{1}, \ldots, x_{n}\right\}$, a set of clauses $\mathcal{C}=\left\{C_{1}, \ldots, C_{m}\right\}$, and a non-negative weight $w(C) \geq 0$ for each clause $C \in \mathcal{C}$. A truth assignment $d$ is an assignment of the value true or false to each variable in $X$, that is $\forall x \in X, d(x) \in\{$ true, false $\}$. The well known NP-hard MAX SAT (resp., MIN SAT) problem is to find an assignment $d^{*}$ that maximizes (resp., minimizes) the total weight of satisfied clauses $W\left(d^{*}\right)$ where $W(d)=\sum_{\{C \mid d \text { satisfies } C\}} w(C)$. The MAX $k$-SAT (resp., MIN $k$-SAT) problem is the restriction to instances where $\sigma(C) \leq k, \forall C \in \mathcal{C}$. The MAX E $k$-SAT (resp., MIN E $k-\mathrm{SAT}$ ) problem is the restriction to instances where $\sigma(C)=k, \forall C \in \mathcal{C}$. In the unweighted version, every clause $C$ has a weight $w(C)=1$. W.l.o.g., we assume that instances are "simple": a variable occurs at most once in a clause and no couple $C=x, C^{\prime}=\bar{x}$ exists. We also make this assumption. We also note $W\left(\mathcal{C}^{\prime}\right)=\sum_{C \in \mathcal{C}^{\prime}} w(C)$ for any $\mathcal{C}^{\prime} \subseteq \mathcal{C}$.

\subsection{A MAX $k$-SAT congestion game}

The system is given by an instance of the weighted MAX $k$-SAT problem where every clause $C \in \mathcal{C}$ is a facility and every variable $x_{i} \in\left\{x_{1}, \ldots, x_{n}\right\}$ is controlled by an independent and selfish player $i \in\{1, \ldots, n\}$. Player $i$ has strategy set

$$
A_{i}=\left\{\left\{C \in \mathcal{C} \mid x_{i} \text { occurs unnegated in } C\right\},\left\{C \in \mathcal{C} \mid x_{i} \text { occurs negated in } C\right\}\right\}
$$

or equivalently, $A_{i}=\{$ true, false $\}$. The system's state (a strategy profile or truth assignment) is denoted by $d=\left(d_{1}, d_{2}, \ldots, d_{n}\right) \in\{\text { true, false }\}^{n}$, where $d_{i}$ is $i$ 's strategy. As in the classical MAX SAT problem, the quality is measured by the total weight of the set of satisfied clauses. The above model is not yet a game because the players have no preference between true and false. We suppose then that the system also guides the players in their choice by giving rewards. To do so, it uses a payment scheme $\mathcal{P}:\left\{\right.$ true, false $^{n} \rightarrow R^{n}$ where $\mathcal{P}_{i}(d)$ is what $i$ receives when $d$ is the strategy profile. Then, every player $i$ rationally acts in order to maximize $\mathcal{P}_{i}(d)$.

We focus on decentralized payment schemes where $\mathcal{P}_{i}(d)$ is defined as a sum of atomic rewards over the set of facilities, i.e., $\mathcal{P}_{i}(d)=\sum_{C \in \mathcal{C}} \pi\left(x_{i}, C, d\right)$ with $\pi\left(x_{i}, C, d\right)$, denoting what $i$ receives from $C$ when $d$ is the system's state, does not depend on the other clauses $\mathcal{C} \backslash C$. The motivation is that such a scheme can be easily implemented in a distributed system since it does not require communication between facilities.

\subsection{Payments and potential functions}

We consider general payment schemes such that $\pi\left(x_{i}, C, d\right)$ is proportional to $w(C)$ : a player satisfying a clause $C$ with $j-1$ others receives $f(j) w(C)$ from $C$ where $f(j) \geq 0$; she receives nothing from $C$ if she does not satisfy it. Here, $f$ is called the payment function. Since $f()$ and $c . f()$ lead to equivalent payment schemes if $c$ is a positive constant, we fix $f(1)=1$.

As it is defined, the MAX $k$-SAT game is a congestion game. We know from $[12,15]$ that it always has a pure Nash equilibrium. Indeed, the game admits a potential function

$$
\Phi(d)=\sum_{C \in \mathcal{C}} \sum_{l=1}^{\nu(d, C)} f(l) w(C)
$$

where $\nu(d, C)$ denotes the number of players satisfying $C$ when $d$ is the strategy profile.

A natural way to share the weight of a clause is to cut it evenly, i.e., $f(j)=1 / j$. As in [3], we will call it the fair payment scheme. Every player $i$ maximizes

$$
\mathcal{P}_{i}(d)=\sum_{C \in \mathcal{C}} \pi\left(x_{i}, C, d\right)=\sum_{j=1}^{k} \frac{1}{j} W\left(\operatorname{cov}_{j}(i, d)\right)
$$


where $\operatorname{cov}_{j}(i, d)$ denotes the set of clauses satisfied by player $i$ and exactly $j-1$ other players. This payment has the following nice property: $\sum_{i=1}^{n} \mathcal{P}_{i}(d)=W(d)$.

In that case, the potential function is

$$
\Phi(d)=\sum_{C \in \mathcal{C}} \sum_{l=1}^{\nu(d, C)} \frac{w(C)}{l}=\sum_{j=1}^{k} H(j) W\left(\operatorname{cov}_{j}(d)\right)
$$

where $H(j)=1+\frac{1}{2}+\frac{1}{3}+\cdots+\frac{1}{j}$ and $\operatorname{cov}_{j}(d)$ denotes the set of clauses satisfied by exactly $j$ variables. Hence, the Nash equilibria of the MAX SAT game with the fair payment function are different from the local optima of the local search algorithm with the 1-neighborhood and the classical economic function $\sum_{j=1}^{k} W\left(\operatorname{cov}_{j}(d)\right)$.

\subsection{Generalities}

W.l.o.g. we will assume along the paper that $d=\left\{\right.$ true $^{n}$ is the worst Nash equilibrium ${ }^{2}$ and $d^{*}$ is an optimal truth assignment. Moreover, $S$ (resp., $S^{*}$ ) will be the set of clauses that $d$ (resp., $d^{*}$ ) satisfies. Thus, $\mathrm{PoA} \geq W(S) / W\left(S^{*}\right)$ (resp., PoA $\leq W(S) / W\left(S^{*}\right)$ ) for the MAX $k$-SAT game (resp., MIN $k$-SAT game). We can write $\pi\left(x_{i}, C\right)$ instead of $\pi\left(x_{i}, C, d\right)$ since $d=\left\{\right.$ true $^{n}$.

Now, we define the notion of atomic gain which will be useful in the proofs.

Definition 1. Given a variable $x \in X$ and a clause $C \in \mathcal{C}$, the atomic gain of $x$ in $C$ under the payment function $f$ is denoted by $\gamma(x, C)$ and defined as follows:

$$
\gamma(x, C)= \begin{cases}0 & \text { when } x \text { does not occur in } C \\ f(j) w(C) & \text { when } C \text { contains the unnegated variable } x \\ -f(j) w(C) & \text { when } C \text { contains } \bar{x} \text { and exactly } j-1 \text { unnegated variables }\end{cases}
$$

Actually, $\gamma(x, C)$ is $x$ 's reward when she satisfies $C$, otherwise this is the opposite of what she would get if she changed her strategy.

\section{The PoA of the MAX $k$-SAT game}

In this section, we consider the MAX $k$-SAT game with the fair payment scheme.

Theorem 1. The PoA of the MAX E $k$-SAT game is $k /(k+1)$, even in the unweighted case.

Proof. Since $d=\{t r u e\}^{n}$ is a NE we have $\sum_{C \in \mathcal{C}} \gamma(x, C) \geq 0$ for any $x \in X$. Summing this inequality over all clauses, we get $\sum_{C \in \mathcal{C}} \sum_{x \in X} \gamma(x, C) \geq 0$ or equivalently,

$$
\sum_{C \in S} \sum_{x \in X} \gamma(x, C) \geq-\sum_{C \in S^{*} \backslash S} \sum_{x \in X} \gamma(x, C)-\sum_{C \in \mathcal{C} \backslash\left(S^{*} \cup S\right)} \sum_{x \in X} \gamma(x, C)
$$

We easily remark that $-\sum_{C \in \mathcal{C} \backslash\left(S^{*} \cup S\right)} \sum_{x \in X} \gamma(x, C) \geq 0$ since each clause $C \notin S^{*} \cup S$ is only compposed of negated variables. Thus, we get

$$
\sum_{C \in S} \sum_{x \in X} \gamma(x, C) \geq-\sum_{C \in S^{*} \backslash S} \sum_{x \in X} \gamma(x, C)
$$

Take any clause $C \in S^{*} \backslash S$. We know that $C$ is composed of $k$ negated variables. We have $\gamma(x, C)=$ $-w(C)$ if $x \in C$ and $\gamma(x, C)=0$ otherwise. Then, $\sum_{x \in X} \gamma(x, C)=-k w(C)$ and the following equality holds.

$$
-\sum_{C \in S^{*} \backslash S} \sum_{x \in X} \gamma(x, C)=\sum_{C \in S^{*} \backslash S} k w(C)=k W\left(S^{*} \backslash S\right)
$$

Now, take any clause $C \in S$. We know that $C$ is composed of $j$ unnegated variables $(1 \leq j \leq k)$ and $k-j$ negated variables. We have $\gamma(x, C)=\frac{1}{j} w(C)$ if $x$ occurs unnegated in $C, \gamma(x, C)=\frac{-1}{j+1} w(C)$ if $x$

\footnotetext{
${ }^{2}$ One can always replace $\bar{x}$ by $x$ and $x$ by $\bar{x}$ in the instance if $d(x)=$ false.
} 
occurs negated in $C$ and $\gamma(x, C)=0$ otherwise. Thus, $\sum_{x \in X} \gamma(x, C)=\left(1-\frac{k-j}{j+1}\right) w(C) \leq w(C)$ and the following inequality holds.

$$
\sum_{C \in S} \sum_{x \in X} \gamma(x, C) \leq \sum_{C \in S} w(C)=W(S)
$$

Using (1), (2) and (3) we get $W(S) \geq k W\left(S^{*} \backslash S\right)$ and thus $W(S) / W\left(S^{*}\right) \geq k /(k+1)$.

We show that $\mathrm{PoA} \leq k /(k+1)$ by considering an instance of the unweighted case with $2 k$ variables $\left\{x_{1}, \ldots, x_{2 k}\right\}$ and $2 k+2$ clauses $\left\{C_{1}, \ldots, C_{2 k+2}\right\}$.

$$
\begin{aligned}
C_{j} & =\bigvee_{l=j-1}^{k+j-2} x_{(l \bmod 2 k)+1} \text { for } 1 \leq j \leq 2 k \\
C_{2 k+1} & =\bigvee_{l=1}^{k} \bar{x}_{l} \\
C_{2 k+2} & =\bigvee_{l=k+1}^{2 k} \bar{x}_{l}
\end{aligned}
$$

It is not difficult to see that $\left\{\right.$ true $^{n}$ is a NE with total weight $2 k$. When only $x_{1}$ and $x_{k+1}$ are set to false then all clauses are satisfied and the optimal weight is $2 k+2$. Thus, $\mathrm{PoA} \leq k /(k+1)$.

Theorem 2. The PoA of the MAX $k$-SAT game is $k /(2 k-1)$, even in the unweighted case.

Proof. Before getting started, we modify the instance in order to characterize $d^{*}$. More precisely, if $X^{+} \subseteq X$ denotes the set of variables appearing only unnegated in $\mathcal{C}$, then we will prove that $d^{*}\left(x_{i}\right)=$ true if $x_{i} \in X^{+}$and $d^{*}\left(x_{i}\right)=$ false otherwise. Moreover, we can suppose that $\mathcal{C}=S \cup S^{*}$.

The transformation is the following: for all $x_{i} \in X$ such that $d^{*}\left(x_{i}\right)=$ true, remove every occurrence of $\overline{x_{i}}$. It is not difficult to see that $d=\left\{\right.$ true $^{n}{ }^{n}$ remains a NE. Moreover $W(S)$ and $W\left(S^{*}\right)$ are unchanged. Actually, $S^{*}$ may not be optimal anymore but the PoA can only be worse. Note that by this process we will get $\mathcal{C} \backslash\left(S \cup S^{*}\right)=\emptyset$. Finally, we always assume that a clause cannot be only composed of unnegated variables of $X^{+}$(the PoA can only be worse if we delete those clauses). Let $\kappa$ be a function defined as follows.

$$
\kappa(x, C)= \begin{cases}\gamma(x, C)-w(C) / k & \text { when } x \in X^{+} \text {and } x \text { occurs in } C \\ \gamma(x, C) & \text { otherwise }\end{cases}
$$

We have

$$
\forall x \in X, \sum_{C \in S^{*} \cup S} \kappa(x, C) \geq 0
$$

Actually, it holds for any variable $x \in X \backslash X^{+}$because $d$ is a NE. Now, every $x \in X^{+}$rationally plays strategy true and her atomic gain is at least $w(C) / k$ in any clause $C$ where she occurs. Summing inequalities (4) over all $x \in X$, we obtain

$$
\sum_{C \in S} \sum_{x \in X} \kappa(x, C) \geq-\sum_{C \in S^{*} \backslash S} \sum_{x \in X} \kappa(x, C)
$$

Let $C$ be a clause of $S^{*} \backslash S$. We know that $C$ is only composed of negated variables belonging to $X \backslash X^{+}$. For every variable $x$ appearing in $C$, we have $\kappa(x, C)=\gamma(x, C)=-w(C)$. Hence, we have $\sum_{x \in X} \kappa(x, C)=-\sigma(C) w(C) \leq-w(C)$ from which we obtain

$$
-\sum_{C \in S^{*} \backslash S} \sum_{x \in X} \kappa(x, C) \geq W\left(S^{*} \backslash S\right)
$$

Now, we prove the following inequality.

$$
\sum_{C \in S} \sum_{x \in X} \kappa(x, C) \leq \frac{k-1}{k} W\left(S \cap S^{*}\right)+W\left(S \backslash S^{*}\right)
$$

Let $C$ be a clause of $S$. If $C$ only contains unnegated variables, i.e., $C=x_{1} \vee \cdots \vee x_{p}$ for some $p \leq k$, then we consider two cases: 
$-C \in S \backslash S^{*}$. All variables in $C$ are set to false by $d^{*}$. Then, they all belong to $X \backslash X^{+}$and $\sum_{x \in X} \kappa(x, C)=\sum_{x \in X} \gamma(x, C)=w(C)$.

$-C \in S \cap S^{*}$. At least one variable in $C$, say $x_{1}$ w.l.o.g., is set to true by $d^{*}$. Then, $x_{1} \in X^{+}$and $\sum_{x \in X} \kappa(x, C) \leq \sum_{x \in X \backslash\left\{x_{1}\right\}} \gamma(x, C)+\kappa\left(x_{1}, C\right) \leq \sum_{x \in X} \gamma(x, C)-\frac{w(C)}{k}=\frac{k-1}{k} w(C)$.

If $C$ contains both unnegated and negated variables, i.e., $C=x_{1} \vee \cdots \vee x_{j} \vee \bar{x}_{j+1} \vee \cdots \vee \bar{x}_{p}$ with $1 \leq j \leq p-1 \leq k-1$, then $\left\{x_{j+1}, \ldots, x_{p}\right\} \subseteq X \backslash X^{+}$. We have $C \in S \cap S^{*}$ and $\sum_{x \in X} \kappa(x, C) \leq \sum_{i=1}^{p} \gamma\left(x_{i}, C\right)-\frac{p-j}{j+1} w(C)=w(C)-\frac{p-j}{j+1} w(C) \leq \frac{k-1}{k} w(C)$.

Inequality (7) holds because $\sum_{x \in X} \kappa(x, C) \leq w(C)$ for all $C \in S \backslash S^{*}$ and $\sum_{x \in X} \kappa(x, C) \leq \frac{k-1}{k} w(C)$ for all $C \in S \cap S^{*}$. Using inequalities (5), (6) and (7), we get $W\left(S^{*} \backslash S\right) \leq \frac{k-1}{k} W\left(S \cap S^{*}\right)+W\left(S \backslash S^{*}\right)$. Thus, $k W\left(S^{*}\right) \leq(2 k-1) W(S)$.

The following instance of the unweighted case shows the tightness of the analysis: $C_{i}=y_{i} \vee x_{1} \vee x_{2} \vee$ $\ldots \vee x_{k-1}$ for $i=1, \ldots, k$, and $C_{k+i}=\overline{x_{i}}$ for $i=1, \ldots, k-1$.

Corollary 1. The price of anarchy of the MAX SAT game is $1 / 2$ with the fair payment scheme, even in the unweighted case.

In the next section, we modify $f$ in order to get a better PoA.

\section{The PoA with non oblivious payment functions}

First, we analyze a parameterized payment function for the MAX E 2 - SAT game where $f(1)=1$ and $f(2)=\varepsilon .^{3}$

Theorem 3. The PoA of the MAX E 2 - SAT game is $\frac{2}{3-\varepsilon}$ if $\varepsilon \in[0 ; 1 / 3]$ and $\frac{1}{1+\varepsilon}$ if $\varepsilon \in[1 / 3 ; 1]$. Moreover, these ratios are tight even in the unweighted case.

Proof. We first remark that inequalities (1) and (2) remain valid with the non oblivious payment scheme under consideration. Take any clause $C \in S$ :

- If $C=x \vee y$ then $\sum_{x \in X} \gamma(x, C)=2 \varepsilon w(C)$.

- If $C=x \vee \bar{y}$ then $\sum_{x \in X} \gamma(x, C)=(1-\varepsilon) w(C)$.

In conclusion, we have:

$$
\sum_{C \in S} \sum_{x \in X} \gamma(x, C) \leq \max \{2 \varepsilon ; 1-\varepsilon\} W(S)
$$

Using (1), (2) and (8) we get:

$$
\max \{2 \varepsilon ; 1-\varepsilon\} W(S) \geq 2 W\left(S^{*} \backslash S\right)
$$

which, with simple algebra gives

$$
W(S) / W\left(S^{*}\right) \geq 2 / \max \{2+2 \varepsilon ; 3-\varepsilon\}
$$

Thus, if $\varepsilon \in[0 ; 1 / 3], W(S) / W\left(S^{*}\right) \geq 2 /(3-\varepsilon)$ and if $\varepsilon \in[1 / 3 ; 1]$ we have $W(S) / W\left(S^{*}\right) \geq 1 /(1+\varepsilon)$. For the tightness, we consider two instances:

$-\varepsilon \in[0 ; 1 / 3]$. We have $C_{1}=x \vee \bar{y}, C_{2}=\bar{x} \vee y$ and $C_{3}=\bar{x} \vee \bar{y}$ with $w\left(C_{1}\right)=w\left(C_{2}\right)=1$ and $w\left(C_{3}\right)=1-\varepsilon$. It is not difficult to see that the PoA is $2 /(3-\varepsilon)$.

$-\varepsilon \in[1 / 3 ; 1]$. We have $C_{1}=x \vee y$ and $C_{2}=\bar{x} \vee \bar{y}$ with $w\left(C_{1}\right)=1$ and $w\left(C_{2}\right)=\varepsilon$. It is not difficult to see that the $\mathrm{PoA}$ is $1 /(1+\varepsilon)$.

\footnotetext{
$\overline{{ }^{3} \text { We restrict }}$ ourselves to $0 \leq \varepsilon \leq 1$ because a simple instance shows that the PoA $\leq 1 / 2$ if $\varepsilon \geq 1: x \vee y$ and $\bar{x} \vee \bar{y}$.
} 
Tight unweighted instances also exist. Assume that $\varepsilon$ is a rational $p / q$. If $q \geq 3 p$ (or equivalently $\varepsilon \leq 1 / 3)$, we consider the following instance: $C_{1, i, j}^{\ell}=x_{i}^{\ell} \vee \bar{a}_{j}^{\ell}$ and $C_{2, i, j}^{\ell}=y_{i}^{\ell} \vee \bar{b}_{j}^{\ell}$ with $\ell, j=1, \ldots, p$ and $i=1, \ldots,(q-p), C_{3, i, j}^{\ell}=b_{i}^{\ell} \vee \bar{a}_{j}^{\ell}$ and $C_{4, i, j}^{\ell}=a_{i}^{\ell} \vee \bar{b}_{j}^{\ell}$ with $i, j, \ell=1, \ldots, p$ and finally $C_{5, i, j}^{\ell}=\bar{x}_{\ell}^{i} \vee \bar{y}_{\ell}^{j}$ with $\ell=1, \ldots,(q-p)$ and $i, j=1, \ldots, p$.

It is not difficult to see that $d=\{\text { true }\}^{n}$ is a NE and $d^{*}=\{f a l s e\}^{n}$ satisfies all clauses. We have $W(d)=2 q p^{2}$ and $W\left(d^{*}\right)=2 q p^{2}+p^{2}(q-p)$; thus $\operatorname{PoA}=2 /(3-\varepsilon)$.

If $p / q=\varepsilon \geq 1 / 3$, we consider the following instance: $C_{1, i, j}^{\ell}=x_{i}^{\ell} \vee y_{j}^{\ell}$ with $\ell=1, \ldots, p$ and $i, j=$ $1, \ldots, q$, and $C_{2, i, j}^{\ell}=\bar{x}_{\ell}^{i} \vee \bar{y}_{\ell}^{j}$ with $\ell=1, \ldots, q$ and $i, j=1, \ldots, p$. It is not difficult to see that $d=\left\{\right.$ true $^{n}$ is a NE. All clauses are satisfied if $x$-variables are set to true and $y$-variables are set to false; we denote this optimal assignment by $d^{*}$. We get $W(d)=p q^{2}$ and $W\left(d^{*}\right)=p q^{2}+q p^{2}$; thus $P o A=1 /(1+\varepsilon)$.

Using Theorem 3, we deduce that $\varepsilon=1 / 3$ gives the best payment scheme for the MAX E $2-$ SAT game and the corresponding PoA is $3 / 4$. The potential function of the game is $\Phi(d)=W\left(S_{1}\right)+\frac{4}{3} W\left(S_{2}\right)$ where $W\left(S_{i}\right)$ is the weight of clauses satisfied by $i$ literals, $i=1,2$. The correspondence with non oblivious local search is now clear since Khanna et al [10] use $\frac{3}{2} W\left(S_{1}\right)+2 W\left(S_{2}\right)=\frac{3}{2} \Phi(d)$ to guide their local search procedure.

We can undertake the same analysis for the MAX E $k$-SAT game but the resulting expression is not simple. Thus, we directly propose the function leading to the best PoA: when exactly $j$ variables satisfy a clause $C$, each of them receives $\alpha_{j}^{\sigma(C)} w(C)$ where $\sigma(C)$ is the size of $C$. Here, $\left(\alpha_{j}^{k}\right)_{j=1 . . k}$ is a sequence defined by $\alpha_{j}^{k}=\frac{k}{j\left(2^{k}-1\right)}+\frac{(k-j)}{j} \alpha_{j+1}^{k}$ which satisfies $\alpha_{k}^{k}=\frac{1}{2^{k}-1}$ and $\alpha_{1}^{k}=1$. The atomic gain $\gamma(x, C)$ is 0 when $x$ does not appear in $C, \alpha_{j}^{\sigma(C)} w(C)$ when $C$ contains the unnegated variable $x$ and exactly $j-1$ other unnegated variables, and $-\alpha_{j}^{\sigma(C)} w(C)$ when $C$ contains $\bar{x}$ and exactly $j-1$ unnegated variables.

Theorem 4. The PoA of the MAX E $k$ - SAT game with the non oblivious payment scheme is $1-1 / 2^{k}$, even in the unweighted case.

Proof. We first remark that inequalities (1) and (2) remain valid with the non oblivious payment scheme.

Take any clause $C \in S$. We know that $C$ is composed of $j$ unnegated variables $(1 \leq j \leq k)$ and $k-j$ negated variables. We have $\gamma(x, C)=\alpha_{j}^{k} w(C)$ if $x$ occurs unnegated in $C, \gamma(x, C)=$ $-\alpha_{j+1}^{k} w(C)$ if $x$ occurs negated in $C$ and $\gamma(x, C)=0$ otherwise. When $C$ contains $k$ unnegated variables, $\sum_{x \in X} \gamma(x, C)=k \alpha_{k}^{k} w(C)=\frac{k}{2^{k}-1} w(C)$. When $C$ contains $j$ unnegated variables and $k-j$ negated variables, $\sum_{x \in X} \gamma(x, C)=\left(j \alpha_{j}^{k}-(k-j) \alpha_{j+1}^{k}\right) w(C)=\frac{k}{2^{k}-1} w(C)$. Thus, we always have $\sum_{x \in X} \gamma(x, C)=\frac{k}{2^{k}-1} w(C)$ and the following equality holds.

$$
\sum_{C \in S} \sum_{x \in C} \gamma(x, C)=\sum_{C \in S} \frac{k}{2^{k}-1} w(C)=\frac{k}{2^{k}-1} W(S)
$$

Using (1), (2) and (9) we get

$$
\frac{k}{2^{k}-1} W(S) \geq k W\left(S^{*} \backslash S\right)
$$

and then,

$$
W(S) / W\left(S^{*}\right) \geq\left(2^{k}-1\right) /\left(2^{k}\right)
$$

We refer to Remark 1 for the tightness.

Interestingly, the proposed non oblivious payment scheme gives the best possible PoA for the MAX E $k$ - SAT game.

Remark 1. There exists a family of instances of the (unweighted) MAX E $k-$ SAT game such that PoA= $1-1 / 2^{k}$ if the payment function is as defined in Subsection 2.2.

Proof. Consider the following (unweighted) instance of the MAX E 2- SAT game:

$$
\begin{aligned}
& \bar{x}_{1} \vee \bar{x}_{2} \\
& x_{1} \vee \bar{x}_{3} \\
& x_{2} \vee \bar{x}_{4} \\
& x_{3} \vee x_{4}
\end{aligned}
$$


It is not difficult to see that $d=\{\text { true }\}^{4}$ is a NE for any payment function $f$ since

$$
\begin{aligned}
& \mathcal{P}_{i}(d)=\mathcal{P}_{i}\left(\left(d_{-i}, \text { false }\right)\right)=f(1), \quad i=1,2 \\
& \mathcal{P}_{i}(d)=\mathcal{P}_{i}\left(\left(d_{-i}, \text { false }\right)\right)=f(2), \quad i=3,4
\end{aligned}
$$

This NE satisfies 3 clauses while $W(($ false,true, false,true $))=4$. Thus, the PoA of the MAX E $2-$ SAT game with any payment function $f$ is bounded by $3 / 4$. We generalize this instance to any $k$ in order to show that the PoA of the MAX E $k$ - SAT game with any payment function $f$ is bounded by $1-1 /\left(2^{k}\right)$.

Let $I_{k}=(X, \mathcal{C})$ be an instance of MAX E $k-$ SAT where $|X|=k 2^{k-1}$ and $|\mathcal{C}|=2^{k}$. Each variable $x \in X$ occurs exactly one time negated and one time unnegated in $I_{k}$. The set of clauses $\mathcal{C}$ is decomposed into $k+1$ clusters $\mathcal{C}_{i}$ for $i=0, \ldots, k$. Each clause of $\mathcal{C}_{i}$ contains $i$ unnegated variables and $k-i$ negated variables. Variables used in cluster $\mathcal{C}_{i}$ are all distinct and finally, the negated variables of cluster $\mathcal{C}_{i}$ appear unnegated in cluster $\mathcal{C}_{i+1}$.

Formally, we have $X=\left\{x_{i, j}: i=0, \ldots, k-1, j=1, \ldots,(k-i)\left(\begin{array}{l}k \\ i\end{array}\right)\right\}$ and $\mathcal{C}=\left\{C_{i, j}: i=0, \ldots, k\right.$ $\left.j=1, \ldots,\left(\begin{array}{l}k \\ i\end{array}\right)\right\}$. Moreover, $\mathcal{C}_{i}=\left\{C_{i, j}: j=1, \ldots,\left(\begin{array}{l}k \\ i\end{array}\right)\right\}$ and we have for $i=0, \ldots, k, j=1, \ldots,\left(\begin{array}{l}k \\ i\end{array}\right)$ :

$$
C_{i, j}=\left(\bigvee_{p=1}^{i} x_{i-1, i(j-1)+p}\right) \vee\left(\bigvee_{p=1}^{k-i} \bar{x}_{i,(k-i)(j-1)+p}\right)
$$

Since $(k-i)\left(\begin{array}{l}k \\ i\end{array}\right)=(i+1)\left(\begin{array}{l}k \\ i+1\end{array}\right)=k\left(\begin{array}{l}k-1 \\ i\end{array}\right)$, the clauses are well defined. Now, $x_{i, j}=$ true is Nash for all non-oblivious payments and it satisfies $2^{k}-1$ clauses (all except $C_{0,1}$ ) whereas $x_{i, j}=$ false if $j=1$ and $x_{i, j}=$ true otherwise satisfies all the $2^{k}$ clauses.

Now, we analyze the MAX $2-$ SAT game when $f(1)=1$ and $f(2)=\varepsilon$.

Theorem 5. The PoA of the MAX 2- SAT game is $\frac{1}{2-\varepsilon}$ if $\varepsilon \in[0 ; 1 / 2]$ and $\frac{1}{1+\varepsilon}$ if $\varepsilon \in[1 / 2 ; 1]$. Moreover, these ratios are tight even in the unweighted case.

Proof. We suppose that the transformation described at the beginning of the proof of Theorem 2 is done. Hence, a clause cannot be only composed of unnegated variables of $X^{+}$. Moreover, $d^{*}(x)=\operatorname{true}$ if $x \in X^{+}$ and $d^{*}(x)=$ false, otherwise. Let $\kappa(x, C)$ be defined by:

$$
\kappa(x, C)= \begin{cases}\gamma(x, C)-\varepsilon w(C) & \text { when } x \in X^{+} \text {and } x \text { occurs in } C \\ \gamma(x, C) & \text { otherwise }\end{cases}
$$

We remark that inequalities (5) and (6) remain valid. Now, take any clause $C \in S$ :

$-C=x$. We know that $x \notin X^{+}$by the previous assumption. Hence, $C \in S \backslash S^{*}$ and $\sum_{x \in X} \kappa(x, C)=$ $w(C)$.

$-C=x \vee y$. If $x \in X^{+}$or $y \in X^{+}, C \in S \cap S^{*}$ then $\sum_{x \in X} \kappa(x, C)=\varepsilon w(C)$ since $x$ and $y$ cannot be both in $X^{+}$. If neither $x$ nor $y$ are in $X^{+}$, we know that $C \in S \backslash S^{*}$ and $\sum_{x \in X} \kappa(x, C)=2 \varepsilon w(C)$.

$-C=x \vee \bar{y}$. We have $C \in S \cap S^{*}$ and $\sum_{x \in X} \kappa(x, C) \leq(1-\varepsilon) w(C)$.

In conclusion, we get:

$$
\sum_{C \in S} \sum_{x \in X} \kappa(x, C) \leq \max \{2 \varepsilon ; 1\} W\left(S \backslash S^{*}\right)+\max \{\varepsilon ; 1-\varepsilon\} W\left(S \cap S^{*}\right)
$$

Using (5), (6) and (10) we get

$$
\max \{2 \varepsilon ; 1\} W\left(S \backslash S^{*}\right)+\max \{\varepsilon ; 1-\varepsilon\} W\left(S \cap S^{*}\right) \geq W\left(S^{*} \backslash S\right)
$$

which implies

$$
W(S) / W\left(S^{*}\right) \geq 1 / \max \{1+\varepsilon ; 2-\varepsilon\}
$$

We propose tight instances which are similar to those given in the proof of Theorem 3 . If $\varepsilon \geq 1 / 2$, see the instances given in the proof of Theorem 3 (case $\varepsilon \geq 1 / 3$ ). If $\varepsilon \leq 1 / 2$, take the instances given in the proof of Theorem 3 (case $\varepsilon \leq 1 / 3$ ) and remplace each clause $C$ composed of two negated variables by two new ones having the first (resp. second) literal of $C$ (the weight remains the same). For $k=2$, we get $C_{1}=x \vee \bar{y}, C_{2}=\bar{x} \vee y$ with $w\left(C_{1}\right)=w\left(C_{2}\right)=1$ and $C_{3}=\bar{x}, C_{4}=\bar{y}$ with $w\left(C_{3}\right)=w\left(C_{4}\right)=1-\varepsilon$.

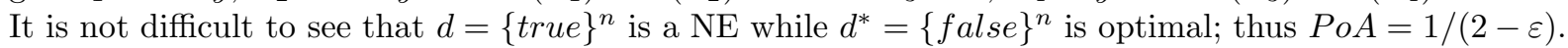


As opposed to the MAX E 2- SAT game, the best PoA for the MAX 2- SAT game is obtained when $\varepsilon=1 / 2$ which corresponds to the fair payment scheme. Actually, we can show the following result.

Theorem 6. The PoA of the MAX $k$-SAT game is $3 / 5$ if $f(j)=\alpha_{j}^{k}$, even in the unweighted case.

Proof. We first apply the modifications of the instance described at the beginning of the proof of Theorem 2. Let $\kappa$ be a function defined as follows:

$$
\kappa(x, C)= \begin{cases}\gamma(x, C)-\alpha_{\sigma(C)}^{\sigma(C)} w(C) & \text { when } x \in X^{+} \text {and } x \text { occurs in } C \\ \gamma(x, C) & \text { otherwise }\end{cases}
$$

We remark that inequalities (5) and (6) still hold. Now, we prove the following inequality.

$$
\sum_{C \in S} \sum_{x \in X} \kappa(x, C) \leq \frac{2}{3} W\left(S \cap S^{*}\right)+W\left(S \backslash S^{*}\right)
$$

Let $C$ be a clause of $S$. If $C$ only contains unnegated variables, i.e., $C=x_{1} \vee \cdots \vee x_{p}$ for some $p \leq k$, then we consider two cases:

$-C \in S \backslash S^{*}$. All variables in $C$ are set to false by $d^{*}$. Then, they all belong to $X \backslash X^{+}$and $\sum_{x \in X} \kappa(x, C)=\sum_{x \in X} \gamma(x, C) \leq w(C)$.

$-C \in S \cap S^{*}$. At least one variable in $C$, say $x_{1}$ w.l.o.g., is set to true by $d^{*}$. This means $x_{1} \in X^{+}$. Moreover, we know that $\sigma(C) \geq 2$ since otherwise $C$ will be only constituted by variables of $X^{+}$. We have $\sum_{x \in X} \kappa(x, C) \leq \sum_{x \in X} \gamma(x, C)-\alpha_{\sigma(C)}^{\sigma(C)} w(C)=(\sigma(C)-1) \alpha_{\sigma(C)}^{\sigma(C)} \leq \frac{2}{3} w(C)$.

If $C$ contains unnegated and negated variables, i.e., $C=x_{1} \vee \cdots \vee x_{j} \vee \bar{x}_{j+1} \vee \cdots \vee \bar{x}_{p}$ with $1 \leq j \leq$ $p-1 \leq k-1$, then $\left\{x_{j+1}, \ldots, x_{p}\right\} \subseteq X \backslash X^{+}$. We have $C \in S \cap S^{*}$ and $\sum_{x \in X} \kappa(x, C)=\sum_{i=1}^{j} \kappa\left(x_{i}, C\right)+$ $\sum_{i=j+1}^{p} \kappa\left(x_{i}, C\right) \leq \sum_{i=1}^{j} \gamma\left(x_{i}, C\right)-(\sigma(C)-j) \alpha_{j+1}^{\sigma(C)} w(C) \leq j \alpha_{j}^{\sigma(C)} w(C)-(\sigma(C)-j) \alpha_{j+1}^{\sigma(C)} w(C)=$ $\frac{\sigma(C)}{2^{\sigma(C)}-1} w(C) \leq \frac{2}{3} w(C)$.

Inequality (11) holds because $\sum_{x \in X} \kappa(x, C) \leq w(C)$ when $C \in S \backslash S^{*}$ and $\sum_{x \in X} \kappa(x, C) \leq \frac{2}{3} w(C)$ when $C \in S \cap S^{*}$.

Using inequalities (5), (6) and (11), we get $W\left(S^{*} \backslash S\right) \leq \frac{2}{3} W\left(S \cap S^{*}\right)+W\left(S \backslash S^{*}\right)$ which, with simple algebra, gives $3 W\left(S^{*}\right) \leq 5 W(S)$.

Since the MAX $k$ - SAT game contains the MAX $2-$ SAT game, we refer to Theorem 5 for the tightness.

We now propose a sequence $\widetilde{\alpha}_{j}^{k}$ leading to the best possible payment scheme (according to the definition of Subsection 2.2) for the MAX $k$-SAT game: $\widetilde{\alpha}_{1}^{k}=1$ and $\widetilde{\alpha}_{j}^{k}=\frac{1}{2(\sigma(C)-1)}$ for $j=2, \ldots, k$.

Theorem 7. The PoA of the MAX $k$ - SAT game with payment function $f(j)=\widetilde{\alpha}_{j}^{k}$ is $2 / 3$, even in the unweighted case.

Proof. We first modify the instance as done in the proof of Theorem 2 and use a function $\kappa$ defined as follows:

$$
\kappa(x, C)= \begin{cases}\gamma(x, C)-\frac{1}{2(\sigma(C)-1)} & \text { when } x \in X^{+} \text {and } x \text { occurs in } C \\ \gamma(x, C) & \text { otherwise }\end{cases}
$$

We remark that inequalities (5) and (6) still hold. Now, we prove the following inequality:

$$
\sum_{C \in S} \sum_{x \in X} \kappa(x, C) \leq \frac{1}{2} W\left(S \cap S^{*}\right)+W\left(S \backslash S^{*}\right)
$$

Let $C$ be a clause of $S$. If $C$ only contains unnegated variables, i.e., $C=x_{1} \vee \cdots \vee x_{p}$ for some $p \leq k$, then we consider two cases:

$-C \in S \backslash S^{*}$. All variables in $C$ are set to false by $d^{*}$. Then, they all belong to $X \backslash X^{+}$and $\sum_{x \in X} \kappa(x, C)=\sum_{x \in X} \gamma(x, C) \leq w(C)$. 
$-C \in S \cap S^{*}$. At least one variable in $C$, say $x_{1}$ w.l.o.g., is set to true by $d^{*}$. This means $x_{1} \in X^{+}$. Moreover, we know that $p \geq 2$ (otherwise, $C$ will be only composed of variables of $X^{+}$). We have $\sum_{x \in X} \kappa(x, C) \leq \frac{p}{2(p-1)} w(C)-\frac{1}{2(p-1)} w(C)=\frac{1}{2} w(C)$.

If $C$ contains both unnegated and negated variables, i.e., $C=x_{1} \vee \cdots \vee x_{j} \vee \bar{x}_{j+1} \vee \cdots \vee \bar{x}_{p}$ with $1 \leq j \leq p-1 \leq k-1$, then $\left\{x_{j+1}, \ldots, x_{p}\right\} \subseteq X \backslash X^{+}$. Thus, we have $C \in S \cap S^{*}$.

If $j=1$, then $\sum_{x \in X} \kappa(x, C)=\left(1-\frac{p-1}{2(p-1)}\right) w(C)=\frac{1}{2} w(C)$. Otherwise, $j \geq 2$ and then $p \geq 3$. We have $\sum_{x \in X} \kappa(x, C)=\left(\frac{j}{2(p-1)}-\frac{p-j}{2(p-1)}\right) w(C) \leq \frac{p-2}{2(p-1)} w(C) \leq \frac{1}{2} w(C)$.

Inequality (12) holds because $\sum_{x \in X} \kappa(x, C) \leq w(C)$ whenever $C \in S \backslash S^{*}$ and $\sum_{x \in X} \kappa(x, C) \leq \frac{1}{2} w(C)$ whenever $C \in S \cap S^{*}$.

Using inequalities (5), (6) and (12), we get $W\left(S^{*} \backslash S\right) \leq \frac{1}{2} W\left(S \cap S^{*}\right)+W\left(S \backslash S^{*}\right)$ which, with simple calculus, gives $2 W\left(S^{*}\right) \leq 3 W(S)$.

Finally, consider the following instance of the unweighted MAX $k-$ sAT problem: $C_{1}=\bar{x}, C_{2}=x \vee \bar{y}$ and $C_{3}=y \vee z$. When each variable plays true then they are at a NE satisfying two clauses, whatever how $f$ is defined. If $x$ and $y$ play false while $z$ plays true, three clauses are satisfied. Thus, the non oblivious payment function given here is the best possible.

Corollary 2. The PoA of the MAX SAT game is $2 / 3$ with the non oblivious payment scheme, even in the unweighted case.

Corollary 3. There exists a polynomial time local search algorithm with locality gap $2 / 3$ for the unweighted MAX $-k$ SAT problem.

Proof. Use the potential function w.r.t. the non oblivious payment $\widetilde{\alpha}_{j}^{k}$ as economic function and the 1-neighborhood: $\Phi(\tau)=\sum_{j=1}^{m} \frac{2 k+j-3}{2 k-2} \operatorname{cov}_{j}(\tau)$.

\section{The PoS of the MAX $k$-SAT game}

We investigate the price of stability [3] of the MAX SAT game and present non trivial instances in which each player possesses a strictly dominant strategy (a strategy strictly better than the others, whatever the other players choose). In such instances, only one NE exists, hence PoA=PoS. We restrict ourselves to the fair and non oblivious payment schemes; however there exists a payment scheme with $\mathrm{PoS}=1 .{ }^{4}$

Theorem 8. The price of stability of the MAX E $k$ - SAT game with the fair payment scheme is $k /(k+1)$ for large enough instances.

Proof. A special instance $I_{p}=(X, \mathcal{C})$ of the MAX E $k$ - SAT game with the fair payment can be built as follows:

Let the variables set be $X=X_{i}^{j} \cup T, \forall i \in\{1, \ldots, k\}, j \in\{1, \ldots, k\}$ with $X_{i}^{j}=\left\{x_{(i-1) p+1}^{j}, \ldots, x_{i p}^{j}\right\}$ and $T=\left\{t_{1}, \ldots, t_{k-1}\right\}$. We note $\bar{X}=\{\bar{x} x \in X\}$ and $(X, Y, \ldots, Z)=\{x \vee y \ldots \vee z: x \in X, y \in Y, \ldots, z \in Z\}$.

The clause set $\mathcal{C}$ consists of the following four parts:

$$
\begin{gathered}
\left(\bar{X}_{i}^{1}, \cdots, \bar{X}_{i}^{k}\right), i \in\{1, \ldots, k\} \\
\left(X_{j}^{1}, \cdots, X_{i}^{k}\right), i, j \in\{1, \ldots, k\} \\
\left\{x_{(i-1) k+1}^{j} \vee \cdots \vee x_{i k}^{j} \mid i \in\{1, \ldots, p\}, j \in\{1, \ldots, k-1\}\right\} \\
\left(\left\{t_{1}\right\}, \ldots,\left\{t_{k-1}\right\}, X_{i}^{k}\right), i \in\{1, \ldots, k\}
\end{gathered}
$$

Clearly, $d=\left\{\right.$ true $^{n}$ where $n=|X|$ is dominant strategy with the fair payment. Indeed,

\footnotetext{
${ }^{4}$ Set $f(1)=1$ and $f(i)=0$, for $i \neq 1$. The potential function $\Phi(d)$ is $\sum_{j=1}^{m} W\left(\operatorname{cov}_{j}(d)\right)$. Hence, any NE - a local optimum w.r.t. $\Phi-$ is a global optimum.
} 
- for every $x \in X_{i}^{j}, i, j \in\{1, \ldots, k\}$ the corresponding positive literal occurs in $k p^{k-1}$ clauses of type (14) plus one clause of type (15) for $j \in\{1, \ldots, k-1\}$ and one clause of type (16) for $j=k$; hence if set to true it gets payoff at least $\frac{k p^{k-1}+1}{k}$; if set to false it gets payoff at most the number of clauses in which the corresponding negative literal $\bar{x}$ occurs (all of type (13)), i.e., $p^{k-1}$;

- finally, for every $t \in T$, the corresponding positive literal occurs in $k p$ clauses of type (16); hence if set to true it gets payoff at least $p$; if set to false, it gets zero payoff, since there is no occurrence of its corresponding negative literal in any clause.

Thus, for the above instance there is a unique Nash Equilibrium, in which all clauses of type (14), (15), (16) are satisfied, i.e., in total $k^{2} p^{k}+(2 k-1) p$ clauses.

However, all $k(k+1) p^{k}+(2 k-1) p$ clauses of the instance can be satisfied, for example by setting all $z \in X_{i}^{k}, i \in\{1, \ldots, k\}$ to false while letting all the rest be set to true.

Combining the two previous propositions we get finally

$$
\operatorname{PoS}\left(I_{p}\right)=\frac{k^{2} p^{k}+(2 k-1) p}{k(k+1) p^{k}+(2 k-1) p}
$$

which tends asymptotically to $\frac{k}{k+1}$ as claimed.

One can remark that the PoS of the MAX $k$ - SAT game is $2 / 3$ when $k=2$ by Theorem 8 .

Theorem 9. The price of stability of the MAX $k-\mathrm{SAT}$ game with the fair payment scheme is at most $\frac{k+1}{2 k}$.

Proof. Just add variable $y_{0}$ and clause $C_{0}=y_{0} \vee x_{1} \vee x_{2} \vee \ldots \vee x_{k-1}$ to the instance given in the proof of Theorem 2. The new instance admits a NE with dominant strategy (everyone plays true) which satisfies $k+1$ clauses. When $x_{i}$ is set to false for $i=1, \ldots, k-1$ and $y_{i}$ is set to true for $i=0, \ldots, k$, the whole set of clauses is satisfied. Since the instance contains $2 k$ clauses, the PoS is at most $\frac{k+1}{2 k}$.

Theorem 10. The price of stability of the MAX E $k$ - SAT game with the non oblivious payment scheme using $f(j)=\alpha_{j}^{k}$ is $1-1 / 2^{k}$ for large enough instances.

Proof. A special instance $I_{p}=(X, \mathcal{C})$ of the MAX E $k$ - SAT game with the non-oblivious payment $\alpha_{i}^{k}$ given before Theorem 4 can be built as follows:

Let the variables set be $X=X_{i}^{j} \cup T, \forall 1 \leq i \leq 3, j \in\{1, \ldots, k\}$ with $X_{i}^{j}=\left\{x_{(i-1) p+1}, \cdots, x_{i p}^{j}\right\}$ for some $p=k q(q \in \mathbb{N})$ and $T=\left\{t_{1}, \cdots, t_{k-1}\right\}$.

Using the same notations as in the previous proof, i.e., $\bar{X}=\{\bar{x} \mid x \in X\}$ and $(X, Y, \cdots, Z)=\{x \vee$ $y \ldots \vee z \mid x \in X, y \in Y, \cdots, z \in Z\}$, the clause set $\mathcal{C}$ can be specified by the following four components (18), (19), (20) and (21):

$$
\begin{gathered}
\left(\bar{X}_{i}^{1}, \cdots, \bar{X}_{i}^{k-1}, \bar{X}_{i}^{k}\right), 1 \leq i \leq 3 \\
\left(X_{i}^{1} \cup X_{j}^{1}, \cdots, X_{i}^{k} \cup X_{j}^{k}\right), i, j \in\{1,2,3\}, i \neq j \\
\left\{x_{(i-1) k+1}^{j} \vee \cdots \vee x_{i k}^{j} \mid i \in\{1, \ldots, q\}\right\}, j \in\{1, \ldots, k-1\} \\
\left(\left\{t_{1}\right\}, \ldots,\left\{t_{k-1}\right\}, X_{i}^{k}\right), 1 \leq i \leq 3
\end{gathered}
$$

Clearly, $d=\left\{\right.$ true $^{n}$ with $n=|X|$ is dominant strategy with the non-oblivious payment $\alpha_{i}^{k}$. Indeed,

- For every $x \in X_{i}^{j}, i, j \in\{1, \ldots, k\}$ the corresponding positive literal occurs in $2(2 p)^{k-1}-p^{k-1}=$ $\left(2^{k}-1\right) p^{k-1}$ clauses of type (19) plus one clause of type (20) for $j \in\{1, \ldots, k-1\}$ and plus one clause of type (21) for $j=k$; hence if set to true it gets payoff at least $p^{k-1}+\frac{1}{2^{k}-1}$; if set to false it gets payoff at most the number of clauses in which the corresponding negative literal $\bar{x}$ occurs (all of type (18)), i.e., $p^{k-1}$

- finally, for every $t \in T$, the corresponding positive literal occurs in $3 p$ clauses (of type (21)), hence if set to true it gets payoff at least $\frac{3 p}{2^{k}-1}$; if set to false, it gets zero payoff, since there is no occurrence of its corresponding negative literal in any clause. 
Thus, for the above instance there is a unique Nash Equilibrium, in which all clauses of type (19), (20), (21) are satisfied, i.e., in total $3\left(2^{k}-1\right) p^{k}+3(k-1) q+3 p$ clauses.

However, all $3 \cdot 2^{k} p^{k}+3(k-1) q+3 p$ clauses of the instance can be satisfied, for example by setting all $z \in X_{i}^{k}, 1 \leq i \leq 3$ to false while letting all the rest be set to "TRUE".

Combining the two previous propositions we get finally

$$
\operatorname{PoS}\left(I_{p}\right)=\frac{3\left(2^{k}-1\right) p^{k}+3(k-1) q+3 p}{3 \cdot 2^{k} p^{k}+3(k-1) q+3 p}
$$

which tends asymptotically to $\frac{2^{k}-1}{2^{k}}$ as claimed.

Since $\widetilde{\alpha}_{j}^{k}$ corresponds to the fair payment scheme when $k=2$ and MAX E $2-$ SAT is a particular case of MAX $k$-SAT, Theorem 8 gives the following result.

Corollary 4. The price of stability of the MAX $k$ - SAT game with the non oblivious payment scheme using $f(j)=\widetilde{\alpha}_{j}^{k}$ is $2 / 3$ for large enough instances.

\section{On a MIN $k$ - SAT game}

We consider the minimization version of the SAT game. Instead of giving rewards, the system uses penalties proportionnal to the weight of the clauses. All variables satisfying a clause evenly share its price/weight. Now, each variable $x_{i}$ tries to minimize $\mathcal{P}_{i}(d)=\sum_{j=1}^{k} \frac{1}{j} W\left(\operatorname{cov}_{j}(i, d)\right)$. We call it the fair penalty scheme.

Any instance with at most $k$ literals per clause can be turned into a new one with exactly $k$ literals per clause such that a NE remains a NE and an optimum remains optimal: just add $k-j$ new negated variables to each clause with $j$ literals. These new variables only appear negated so they must be set to true in any truth assignment at NE. Thus, the MIN $k$ - SAT and the MIN E $k-$ SAT games have the same PoA and PoS.

Let $I$ be the following (unweighted) instance of the MIN $k-$ SAT game: $C_{0}=\bar{x}_{1} \vee \cdots \vee \bar{x}_{k}$ and $C_{i}=x_{i}$ for $i=1, \ldots, k$. The truth assignment $\left\{\right.$ true $^{n}$ is a NE with total weight $k$ while $\{\text { false }\}^{n}$ satisfies only one clause. It shows that the PoA is a least $k$.

Theorem 11. The PoA of the MIN $k$ - SAT and the MIN E $k$ - SAT games with the fair penalty scheme is $k$, even in the unweighted case.

Proof. Let $X^{\prime}=\left\{x \in X \mid d(x) \neq d^{*}(x)\right\}$. We introduce a slightly modified version of $\gamma$ denoted by $\gamma^{\prime}$ and defined by $\gamma^{\prime}(x, C)=0$ if $x \in X \backslash X^{\prime}$, otherwise $\gamma^{\prime}(x, C)=\gamma(x, C)$. We clearly have $\sum_{C \in \mathcal{C}} \gamma^{\prime}(x, C) \leq 0$ for any $x \in X$.

By summing over the variables, we deduce $\sum_{C \in \mathcal{C}} \sum_{x \in X} \gamma^{\prime}(x, C) \leq 0$. Equivalently:

$$
\sum_{C \in S} \sum_{x \in X} \gamma^{\prime}(x, C) \leq-\sum_{C \in S^{*} \backslash S} \sum_{x \in X} \gamma^{\prime}(x, C)-\sum_{C \in \mathcal{C} \backslash\left(S^{*} \cup S\right)} \sum_{x \in X} \gamma^{\prime}(x, C)
$$

We first prove the following:

$$
\sum_{C \in \mathcal{C} \backslash\left(S^{*} \cup S\right)} \sum_{x \in X} \gamma^{\prime}(x, C)=0
$$

Let $C$ be a clause in $\mathcal{C} \backslash\left(S^{*} \cup S\right)$. Since $C \notin S$ and $d=\{\text { true }\}^{n}$, we know that $C$ is only composed of negated variables. So, suppose $C=\bar{x}_{1} \vee \cdots \vee \bar{x}_{p}$ with $p \leq k$. Since $C \notin S^{*}, x_{i} \notin X^{\prime}$ for $i=1, \ldots, p$. Thus, $\gamma^{\prime}(x, C)=0$ for all $x \in X$.

Let $C$ be a clause of $S^{*} \backslash S$. We know that $C$ is only composed of $p$ negated variables. Since $C \in S^{*} \backslash S$ we have $\gamma^{\prime}(x, C) \geq \gamma(x, C)$, and $p \leq k$ then

$$
-\sum_{C \in S^{*} \backslash S} \sum_{x \in X} \gamma^{\prime}(x, C) \leq-\sum_{C \in S^{*} \backslash S} \sum_{x \in X} \gamma(x, C) \leq k W\left(S^{*} \backslash S\right)
$$

Now, we prove the following inequality.

$$
\sum_{C \in S} \sum_{x \in X} \gamma^{\prime}(x, C) \geq W\left(S \backslash S^{*}\right)-\frac{(k-1)}{2} W\left(S \cap S^{*}\right)
$$


Let $C$ be a clause of $S$. Assume that $C=x_{1} \vee \cdots \vee x_{j} \vee \bar{x}_{j+1} \vee \cdots \vee \bar{x}_{p}$ with $1 \leq j \leq p \leq k$, then we consider several subcases:

- For all $r=j+1, \ldots, p, x_{r} \notin X^{\prime}$.

- $C \in S \backslash S^{*}$. All variables $x_{i}$ for $i=1, \ldots, j$ are in $X^{\prime}$. Then, we get $\sum_{x \in X} \gamma^{\prime}(x, C)=\sum_{x \in X} \gamma(x, C)=$ $w(C)$.

- $C \in S \cap S^{*}$ or equivalently, at least one variable in $C$, say $x_{1} \in X^{\prime}$. Since, $\gamma^{\prime}\left(x_{i}, C\right) \geq 0$ for $i=1, \ldots, j$ and $\gamma^{\prime}\left(x_{i}, C\right)=0$ otherwise, we get $\sum_{x \in X} \gamma^{\prime}(x, C) \geq 0 \geq-\frac{(k-1)}{2} w(C)$.

- There exists $x_{r} \in X^{\prime}$ with $j+1 \leq r \leq p$. We deduce $2 \leq j+1 \leq p \leq k$ and $C \in S \cap S^{*}$. Since, $\gamma^{\prime}\left(x_{i}, C\right) \geq 0$ for $i=1, \ldots, j$, and $\gamma^{\prime}\left(x_{i}, C\right) \geq \gamma\left(x_{i}, C\right)$ for $i=j+1, \ldots, p$, we get: $\sum_{x \in X} \gamma^{\prime}(x, C) \geq$ $\sum_{i=j+1}^{p} \gamma\left(x_{i}, C\right)=-\frac{(p-j)}{j+1} w(C) \geq-\frac{(k-1)}{2} w(C)$.

Using inequalities (23), (24), (25) and (26), we get

$$
\begin{aligned}
k W\left(S^{*} \backslash S\right) & \geq W\left(S \backslash S^{*}\right)-\frac{(k-1)}{2} W\left(S \cap S^{*}\right) \\
k W\left(S^{*}\right) & \geq W\left(S \backslash S^{*}\right)+\frac{(k+1)}{2} W\left(S \cap S^{*}\right) \\
k W\left(S^{*}\right) & \geq W\left(S^{*} \cap S\right)+W\left(S \backslash S^{*}\right)=W(S)
\end{aligned}
$$

Using $I$, it is not difficult to see that no penalty scheme, as defined in Subsection 2.2, can improve the PoA of the MIN $k$ - SAT game since when players are at NE $d=\left\{\right.$ true $^{k}$, we have $\forall i \mathcal{P}_{i}(d)=$ $\mathcal{P}_{i}\left(d_{-i}\right.$, false $)=f(1)$.

Let $I^{\prime}$ be the following weighted instance of the MIN $k$-SAT game (an equivalent unweighted instance exists) and consider the fair penalty scheme: $C_{0}=\overline{x_{1}} \vee \cdots \vee \overline{x_{k}}$ with $w\left(C_{0}\right)=1+\varepsilon$ and $C_{i}=x_{i}$ with $w\left(C_{i}\right)=1 / i$ for $i=1, \ldots, k$. If $H(k)>\varepsilon>0$, it is not difficult to see that $d^{*}=\{\text { false }\}^{k}$ is optimal but not at NE. Indeed, if $d=\{\text { false }\}^{k}$ then $x_{k}$ will change her strategy and improve her utility (from $(1+\varepsilon) / k$ to $1 / k)$. After, $x_{k-1}$ will also change her strategy and improve her utility (from $(1+\varepsilon) /(k-1$ ) to $1 /(k-1))$ and so on. Actually, $d=\left\{\right.$ true $^{k}$ is the only NE; its total weight is $1+1 / 2+\cdots+1 / k$. As $\varepsilon$ tends to 0 , the PoS is at best $H(k)$.

Theorem 12. The PoS of the MIN $k$ - SAT game with the fair penalty scheme is $H(k)=1+\frac{1}{2}+\frac{1}{3}+\ldots+\frac{1}{k}$.

Proof. The potential function of the MIN $k$ - SAT game is

$$
\Phi(\tau)=\sum_{j=1}^{k} H(j) W\left(\operatorname{cov}_{j}(\tau)\right)
$$

where $\operatorname{cov}_{j}(\tau)$ denotes the set of clauses satisfied by exactly $j$ literals with the truth assignment $\tau$. If $W(\tau)$ denotes the total weight of $\tau$ then

$$
\forall \tau \in\{\text { true } \text { false }\}^{n}, W(\tau) \leq \Phi(\tau) \leq H(k) W(\tau)
$$

Now, suppose that $d^{*}$ is the initial strategy profile and we let the players play until they reach a NE $d$. Every individual improvement is tracked by $\Phi$, then $\Phi(d) \leq \Phi\left(d^{*}\right)$. We simply use $(27)$ to get $W(d) \leq$ $\Phi(d) \leq \Phi\left(d^{*}\right) \leq H(k) W\left(d^{*}\right)$. The PoS is at most $H(k)$.

\section{Acknowledgment}

We thank Yann Chevaleyre and Nicolas Maudet for useful discussions. 


\section{References}

1. P. Alimonti. New Local Search Approximation Techniques for Maximum Generalized Satisfiability Problems. In Proc. of CIAC 1994, LNCS 778, pp. 40-53, 1994.

2. R. Battiti and M. Protasi. Approximate Algorithms and Heuristics for MAX-SAT. In "Handbook of combinatorial optimization", vol. 1, D.-Z Du and P.M. Pardalos (Eds), pp. 77-148, Kluwer Academic Publishers, 1998.

3. E. Anshelevich, A. Dasgupta, J. M. Kleinberg, É. Tardos, T. Wexler and T. Roughgarden. The Price of Stability for Network Design with Fair Cost Allocation. In Proc. of FOCS 2004, pp. 295-304, 2004.

4. G. Christodoulou and E. Koutsoupias. On the Price of Anarchy and Stability of Correlated Equilibria of Linear Congestion Games. In Proc. of ESA 2005, LNCS 3669, pp. 59-70, 2005.

5. G. Christodoulou and E. Koutsoupias. The Price of Anarchy of Finite Congestion Games. In Proc. of STOC 2005, pp. 67-73, 2005.

6. G. Christodoulou, E. Koutsoupias and A. Nanavati. Coordination Mechanisms. In Proc. of ICALP 2004, LNCS 3142, pp. 345-357, 2004.

7. A. Czumaj and B. Vöcking. Tight bounds for worst-case equilibria. In Proc. of SODA 2002, pp. 413-420, 2002.

8. N. R. Devanur, N. Garg, R. Khandekar, V. Pandit, A. Saberi and V. V. Vazirani. Price of Anarchy, Locality Gap, and a Network Service Provider Game. In Proc. of WINE 2005, LNCS 3828, pp. 1046-1055, 2005.

9. A. Fabrikant, C. H. Papadimitriou and K. Talwar. The complexity of pure Nash equilibria. In Proc. of STOC 2004, pp. 604-612, 2004.

10. S. Khanna, R. Motwani, M. Sudan and U. V. Vazirani. On Syntactic versus Computational Views of Approximability. SIAM Journal on Computing, vol 28(1), pp 164-191, 1998.

11. E. Koutsoupias and C. Papadimitriou. Worst Case Equilibria. In Proc. of STACS 1999, LNCS 1563, pp. 404-413, 1999.

12. D. Monderer and L. S. Shapley. Potential Games. Games and Economic Behavior, vol 14, pp. 124-143, 1996.

13. C. H. Papadimitriou. Algorithms, games, and the internet. In Proc. of STOC 2001, pp. 749-753, 2001.

14. T. Roughgarden and É. Tardos. How bad is selfish routing? In Proc. of FOCS 2000, pp. 93-102, 2000.

15. R. W. Rosenthal. A class of games possessing pure-strategy Nash equilibria. International Journal of Game Theory, vol 2(1), pp. 65-67, 1973. 